EPJ Web of Conferences 95, 01002 (2015)

DOI: $10.1051 /$ epjconf/ 20159501002

C) Owned by the authors, published by EDP Sciences, 2015

\title{
Electromagnetic probes of the QGP
}

\author{
E. L. Bratkovskaya ${ }^{1, a}$, O. Linnyk ${ }^{2}$, and W. Cassing ${ }^{2}$ \\ ${ }^{1}$ Institut für Theoretische Physik, Johann Wolfgang Goethe University Frankfurt/M, Germany \\ ${ }^{2}$ Institut für Theoretische Physik, University of Giessen, Germany
}

\begin{abstract}
We investigate the properties of the QCD matter across the deconfinement phase transition in the scope of the parton-hadron string dynamics (PHSD) transport approach. We present here in particular the results on the electromagnetic radiation, i.e. photon and dilepton production, in relativistic heavy-ion collisions. By comparing our calculations for the heavy-ion collisions to the available data, we determine the relative importance of the various production sources and address the possible origin of the observed strong elliptic flow $v_{2}$ of direct photons. We argue that the different centrality dependence of the hadronic and partonic sources for direct photon production in nucleusnucleus collisions can be employed to shed some more light on the origin of the photon $v_{2}$ "puzzle". While the dilepton spectra at low invariant mass show in-medium effects like an enhancement from multiple baryonic resonance formation or a collisional broadening of the vector meson spectral functions, the dilepton yield at high invariant masses (above $1.1 \mathrm{GeV}$ ) is dominated by QGP contributions for central heavy-ion collisions at ultrarelativistic energies. This allows to have an independent view on the parton dynamics via their electromagnetic massive radiation.
\end{abstract}

\section{Introduction}

The electromagnetic emissivity of strongly interacting matter is a subject of longstanding interest $[1,2]$ and is explored in particular in relativistic nucleus-nucleus collisions, where the photons (and dileptons) measured experimentally provide a time-integrated picture of the collision dynamics. The recent observation by the PHENIX Collaboration [3] that the elliptic flow $v_{2}\left(p_{T}\right)$ of 'direct photons' produced in minimal bias $\mathrm{Au}+\mathrm{Au}$ collisions at $\sqrt{s_{N N}}=200 \mathrm{GeV}$ is comparable to that of the produced pions was a surprise and in contrast to the theoretical expectations and predictions. We will analyse this photon $v_{2}$ "puzzle" within scope of the parton-hadron string dynamics (PHSD) transport approach [4] with a focus on the centrality dependence of the different production sources. Furthermore, the PHSD approach will be used to study dilepton production in nucleus-nucleus collisions from SIS to LHC energies in comparison to available data in order to extract information about the modification of hadron properties in the dense and hot hadronic medium which might shed some light on chiral symmetry restoration (cf. [2] and references therein). On the other hand we intend to identify those spectral regimes where we see a clear dominance of partonic channels that might allow to determine their transport properties via their electromagnetic emissivity.

\footnotetext{
ae-mail: Elena.Bratkovskaya@th.physik.uni-Frankfurt.de
} 


\section{Photon/dilepton emission rates}

In hydrodynamical calculations for the time evolution of the bulk matter the equilibrium emission rate of electromagnetic probes enters which in thermal field theory can be expressed as $[5,6]$ :

$$
q_{0} \frac{d^{3} R}{d^{3} q}=-\frac{g_{\mu v}}{(2 \pi)^{3}} \operatorname{Im} \Pi^{\mu v}\left(q_{0}=|\vec{q}|\right) f\left(q_{0}, T\right)
$$

for photons with 4-momentum $q=\left(q_{0}, \vec{q}\right)$ and

$$
E_{+} E_{-} \frac{d^{3} R}{d^{3} p_{+} d^{3} p_{-}}=\frac{2 e^{2}}{(2 \pi)^{6}} \frac{1}{q^{4}} L_{\mu v} \operatorname{Im} \Pi^{\mu v}\left(q_{0},|\vec{q}|\right) f\left(q_{0}, T\right)
$$

for dilepton pairs with 4-momentum $q=\left(q_{0}, \vec{q}\right)$, where $q=p_{+}+p_{-}$and $p_{+}=\left(E_{+}, \vec{p}_{+}\right), p_{-}=\left(E_{-}, \vec{p}_{-}\right)$. Here the Bose distribution function is $f\left(q_{0}, T\right)=1 /\left(e^{q_{0} / T}-1\right) ; L_{\mu \nu}$ is the electromagnetic leptonic tensor, $\Pi^{\mu v}$ is the retarded photon self-energy at finite temperature $T$ related to the electromagnetic current correlator $\left.\Pi^{\mu v} \sim i \int d^{4} x e^{i p x}\left\langle\left[J_{\mu}(x), J_{v}(0)\right]\right\rangle\right|_{T}$. Using the Vector-Dominance-Model (VDM) $\operatorname{Im} \Pi^{\mu \nu}$ can be related to the in-medium $\rho$-meson spectral function from many-body approaches [7] which, thus, can be probed by dilepton measurements directly. The photon rates for $q_{0} \rightarrow 0$ are related to the electric conductivity $\sigma_{0}$ which allows to probe the electric properties of the QGP [8]. We point out that Eqs.(1),(2) are strictly applicable only for systems in thermal equilibrium whereas the dynamics of heavy-ion collisions is generally of non-equilibrium nature.

The non-equilibrium emission rate from relativistic kinetic theory [6, 9], e.g. for the process $1+2 \rightarrow$ $\gamma+3$, is

$$
q_{0} \frac{d^{3} R}{d^{3} q}=\int \frac{d^{3} p_{1}}{2(2 \pi)^{3} E_{1}} \frac{d^{3} p_{2}}{2(2 \pi)^{3} E_{2}} \frac{d^{3} p_{3}}{2(2 \pi)^{3} E_{3}}(2 \pi)^{4} \delta^{4}\left(p_{1}+p_{2}-p_{3}-q\right)\left|M_{i f}\right|^{2} \frac{f\left(E_{1}\right) f\left(E_{2}\right)\left(1 \pm f\left(E_{3}\right)\right)}{2(2 \pi)^{3}},
$$

where $f\left(E_{i}\right)$ is the distribution function of particle $i=1,2,3$, which can be hadrons (mesons and baryons) or partons. In Eq. (3) $M_{i f}$ is the matrix element of the reaction which has to be evaluated on a microscopical level. In the case of hadronic reactions One-Boson-Exchange models or chiral models are used to evaluate $M_{i f}$ on the level of Born-type diagrams. However, for a consistent consideration of such elementary process in the dense and hot hadronic environment, it is important to account for the in-medium modification of hadronic properties, i.e. many-body approaches such as self-consistent $G$-matrix calculations have to be applied (e.g. [10] for anti-kaons or [7] for $\rho$ mesons).

\section{Photons}

\subsection{Production sources}

There are different production sources of photons in $p+p$ and $A+A$ collisions:

1) Decay photons - most of the photons seen in $p+p$ and $A+A$ collisions stem from the hadronic decays: $m \rightarrow \gamma+X, m=\pi^{0}, \eta, \omega, \eta^{\prime}, a_{1}, \ldots$.

2) Direct photons - obtained by subtraction of the decay photon contributions from the inclusive (total) spectra measured experimentally.

(i) The are a few sources of direct photons at large transverse momentum $p_{T}$ denoted by 'hard' photons: the 'prompt' production from the initial hard $N+N$ collisions and the photons from the jet fragmentation reactions, which are the standard pQCD type of processes. The latter, however, might be modified in $A+A$ contrary to $p+p$ due to the parton energy loss in the medium. 
(ii) At low $p_{T}$ the photons come from the thermalized QGP, so called 'thermal' photons, as well as from hadronic interactions:

- The 'thermal' photons from the QGP arise mainly from $q \bar{q}$ annihilation $(q+\bar{q} \rightarrow g+\gamma)$ and Compton scattering $(q(\bar{q})+g \rightarrow q(\bar{q})+\gamma)$ which can be calculated in leading order pQCD [11]. However, the next-to-leading order corrections turn out to be also important [12].

- Hadronic sources of photons are related to

1) secondary mesonic interactions as $\pi+\pi \rightarrow \rho+\gamma, \rho+\pi \rightarrow \pi+\gamma, \pi+K \rightarrow \rho+\gamma, \ldots$. The binary channels with $\pi, \rho$ have been evaluated in effective field theory [13] and are used in transport model calculations $[14,15]$ within the extension for the off-shellness of $\rho$-mesons due to the broad spectral function. Alternatively, the binary hadron rates (3) have been derived in the massive Yang-Milles approach in Ref. [16] and been often used in hydro calculations .

2) hadronic bremsstrahlung, such as meson-meson $(\mathrm{mm})$ and meson-baryon $(\mathrm{mB})$ bremsstrahlung $m_{1}+m_{2} \rightarrow m_{1}+m_{2}+\gamma, m+B \rightarrow m+B+\gamma$, where $m=\pi, \eta, \rho, \omega, K, K^{*}, \ldots$ and $B=p, \Delta, \ldots$. Here the leading contribution corresponds to the radiation from one charged hadron. The importance of bremsstrahlung contributions to the photon production will be discussed below.

\subsection{Direct photons and the $v_{2}$ 'puzzle'}

The photon production has been measured early in relativistic heavy-ion collisions by the WA98 Collaboration in $\mathrm{S}+\mathrm{Au}$ and $\mathrm{Pb}+\mathrm{Pb}$ collisions at SPS energies [17]. The model comparisons with experimental data show that the high $p_{T}$ spectra are dominated by the hard 'prompt' photon production whereas the 'soft' low $p_{T}$ spectra stem from hadronic sources since the thermal QGP radiation at SPS energies is not large. Moreover, the role of hadronic bremsstrahlung turns out to be very important for a consistent description of the low $p_{T}$ data as has been found a couple of years ago in expanding fireball model calculations [18] and in the HSD (Hadron-String-Dynamics) transport approach [14]. Unfortunately, the accuracy of the experimental data at low $p_{T}$ did not allow to draw further solid conclusions.

The measurement of photon spectra by the PHENIX Collaboration [3] has stimulated a new wave of interest for direct photons from the theoretical side since at RHIC energies the thermal QGP photons have been expected to dominate the spectra. A variety of model calculations based on fireball, Bjorken hydrodynamics, ideal hydrodynamics with different initial conditions and Equations-of-State (EoS) turned out to show substantial differences in the slope and magnitude of the photon spectra (for a model comparison see Fig. 47 of [3] and corresponding references therein). Furthermore, the recent observation by the PHENIX Collaboration [19] that the elliptic flow $v_{2}\left(p_{T}\right)$ of 'direct photons' produced in minimal bias $\mathrm{Au}+\mathrm{Au}$ collisions at $\sqrt{s_{N N}}=200 \mathrm{GeV}$ is comparable to that of the produced pions was a surprise and in contrast to the theoretical expectations and predictions. Indeed, the photons produced by partonic interactions in the quark-gluon plasma phase have not been expected to show a considerable flow because - in a hydrodynamical picture - they are dominated by the emission at high temperatures, i.e. in the initial phase before the elliptic flow fully develops. Since the direct photon $v_{2}\left(\gamma^{d i r}\right)$ is a 'weighted average' $\left(w_{i}\right)$ of the elliptic flow of individual contributions $i$

$$
v_{2}\left(\gamma^{d i r}\right)=\sum_{i} v_{2}\left(\gamma^{i}\right) w_{i}=\frac{\sum_{i} v_{2}\left(\gamma^{i}\right) N_{i}}{\sum_{i} N_{i}},
$$

a large QGP contribution gives a smaller $v_{2}\left(\gamma^{d i r}\right)$. A sizable photon $v_{2}$ has been observed also by the ALICE Collaboration in $\mathrm{Pb}+\mathrm{Pb}$ collisions at the LHC [20]. None of the theoretical models could describe simultaneously the photon spectra and $v_{2}$ which may be noted as a 'puzzle' for theory. Moreover, the PHENIX and ALICE Collaborations have reported recently the observation of non-zero 
triangular flow $v_{3}$ (see $[21,22]$ ). Thus, the consistent description of the photon experimental data remains a challenge for theory.

\subsection{Transport analysis of the photon $v_{2}$ 'puzzle'}

It is important to stress that state-of-the art hydro models reproduce well the hadronic 'bulk' observables (e.g. rapidity distributions, $p_{T}$ spectra and $v_{2}, v_{3}$ of hadrons). However, in spite of definite improvements of the general dynamics by including the fluctuating initial conditions (IP-Glasma or MC-Glauber type) and viscous effects, the hydro models underestimate the spectra and $v_{2}$ of photons at RHIC and LHC energies. For a recent overview we refer the reader to Ref. [23].

As a 'laboratory' for a detailed theoretical analysis we use the microscopic Parton-Hadron-String Dynamics (PHSD) transport approach [4], which is based on the generalized off-shell transport equations derived in first order gradient expansion of the Kadanoff-Baym equations, and applicable for strongly interacting systems. The approach consistently describes the full evolution of a relativistic heavy-ion collision from the initial hard scatterings and string formation through the dynamical deconfinement phase transition to the strongly-interacting quark-gluon plasma as well as dynamical hadronization and the subsequent interactions in the expanding hadronic phase as in the HSD transport approach [24]. The partonic dynamics is based on the Dynamical Quasi-Particle Model (DQPM), that is constructed to reproduce lattice QCD (1QCD) results for a quark-gluon plasma in thermodynamic equilibrium. The DQPM provides the mean felds for gluons/quarks and their effective 2-body interactions that are implemented in the PHSD (for the details see Ref. [25] and [4, 15]). The PHSD model reproduces a large variety of observables from SPS to LHC energies, e.g. transverse mass and rapidity spectra of charged hadrons, dilepton spectra, collective flow coefficients etc. [4, 15]. Since the QGP radiation in PHSD occurs from the massive off-shell quasi-particles with spectral functions, the corresponding QGP rate has been extended beyond the standard pQCD rate [11] - see Ref. [26].

The result of the PHSD approach [15] for the direct photon $p_{T}$-spectrum at midrapidity for $\mathrm{Au}+\mathrm{Au}$ collisions at $\sqrt{s}=200 \mathrm{GeV}$ is shown in Fig. 1 for different centralities in comparison to the PHENIX data [3]. The upper solid lines give the total direct photon spectra whereas the various lines show the contributions from indidividual channels (see legend). While the 'hard' $p_{T}$ spectra are dominated by the 'prompt' (pQCD) photons, the 'soft' spectra are filled by the 'thermal' sources: the QGP gives up to $50 \%$ of the direct photon yield between 1 and $2 \mathrm{GeV} / \mathrm{c}$ for the most central bin $(0-20 \%)$, a sizable contribution stems from hadronic sources such as meson-meson $(\mathrm{mm})$ and meson-Baryon $(m B)$ bremsstrahlung; the contributions from binary $m m$ reactions are of subleading order. Thus, according to the present PHSD results the $m m$ and $m B$ bremsstrahlung turn out to be an important source of direct photons. We note, that the bremsstrahlung channels are not included in the $\mathrm{mm}$ binary 'HG' rate [16] used in the hydro calculations mentioned above. We stress, that $m m$ and $m B$ bremsstrahlung cannot be subtracted experimentally from the photon spectra and has to be included in theoretical considerations. As has been pointed out earlier its importance for 'soft' photons follows also from the WA98 data at $\sqrt{s}=17.3 \mathrm{GeV}[14,18]$.

However, some words of caution have to be given here related to the uncertainties in the bremsstrahlung channels in the present PHSD results. The implementation of photon bremsstrahlung from hadronic reactions in transport approaches $[14,15]$ is based on the 'soft-photon' approximation (SPA) [6] which implies the factorization of the amplitude for the $a+b \rightarrow a+b+\gamma$ processes to the strong and electromagnetic parts assuming that the radiation from internal lines is negligible and the strong interaction vertex is on-shell. In this case the strong interaction part can be approximated by the on-shell elastic cross section for the reaction $a+b \rightarrow a+b$. Thus, the resulting yield of the bremsstrahlung photons depends on the validity of the SPA for large $p_{T}$ itself and assumptions on the cross sections for the meson-meson and meson-baryon elastic scattering which are little (or not at all) 

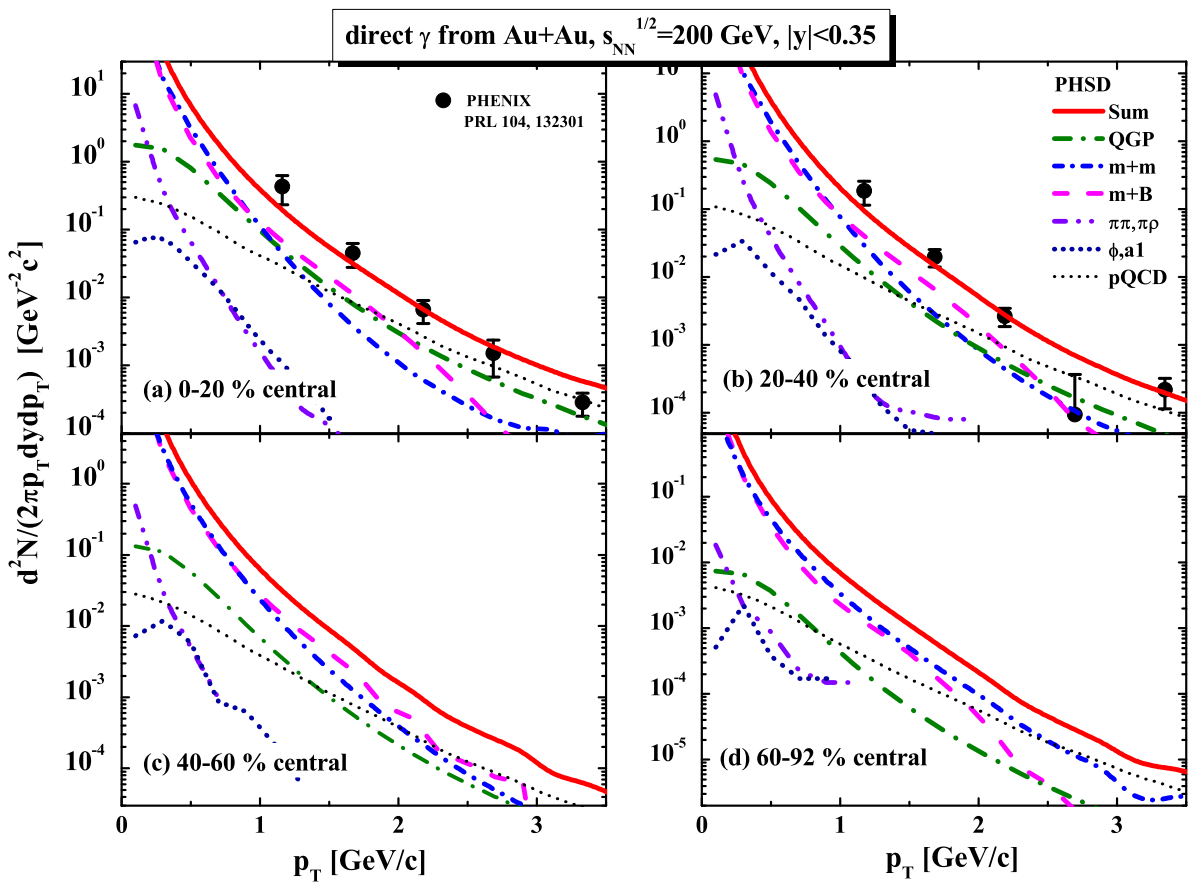

Figure 1. Direct photon $p_{T}$-spectrum from the PHSD approach in comparison to the PHENIX data [3] at midrapidity for different centralities in $\mathrm{Au}+\mathrm{Au}$ collisions at $\sqrt{s_{N N}}=200 \mathrm{GeV}$. The channel description is given in the legend. The figure is taken from Ref. [15].

known experimentally. For a more detailed discussion on uncertainties we refer the reader to Ref. [15]. In this respect we consider the PHSD results on bremsstrahlung as an 'upper estimate'.

The question: "what dominates the photon spectra - QGP radiation or hadronic contributions" can be addressed experimentally by investigating the centrality dependence of the photon yield: the QGP contribution is expected to decrease when going from central to peripheral collisions where the hadronic channels should be dominant. The centrality dependence of the direct photon yield, integrated over different $p_{T}$ ranges, has been measured by the PHENIX Collaboration, too [27, 28]. It has been found that the midrapidity 'thermal' photon yield scales with the number of participants as $d N / d y \sim N_{\text {part }}^{\alpha}$ with $\alpha=1.48 \pm 0.08$ and only very slightly depends on the selected $p_{T}$ range (which is still in the 'soft' sector, i.e. $<1.4 \mathrm{GeV} / c$ ). Note that the 'prompt' photon contribution (which scales as the $p p$ 'prompt' yield times the number of binary collisions in $A+A$ ) has been subtracted from the data. The PHSD predictions [15] for $\mathrm{Au}+\mathrm{Au}$ collisions at different centralities give $\alpha($ total $) \approx 1.5$, which is dominated by hadronic contributions, while the QGP channels scale with $\alpha(Q G P) \sim 1.7$ (see Fig. 2 (1.h.s.)). A similar finding has been obtained by the viscous $(2+1) \mathrm{D}$ VISH2 +1 and $(3+1) \mathrm{D}$ MUSIC hydro models [29]: $\alpha(H G) \sim 1.46, \alpha(Q G P) \sim 2, \alpha($ total $) \sim 1.7$. Thus, the QGP photons show a centrality dependence significantly stronger than that of hadron-gas (HG) photons.

In Fig. 2 (r.h.s.) we provide predictions for the centrality dependence of the direct photon elliptic flow $v_{2}\left(p_{T}\right)$ within the PHSD approach. The direct photon $v_{2}$ is seen to be larger in the peripheral collisions compared to the most central ones. The predicted centrality dependence of the direct photon 

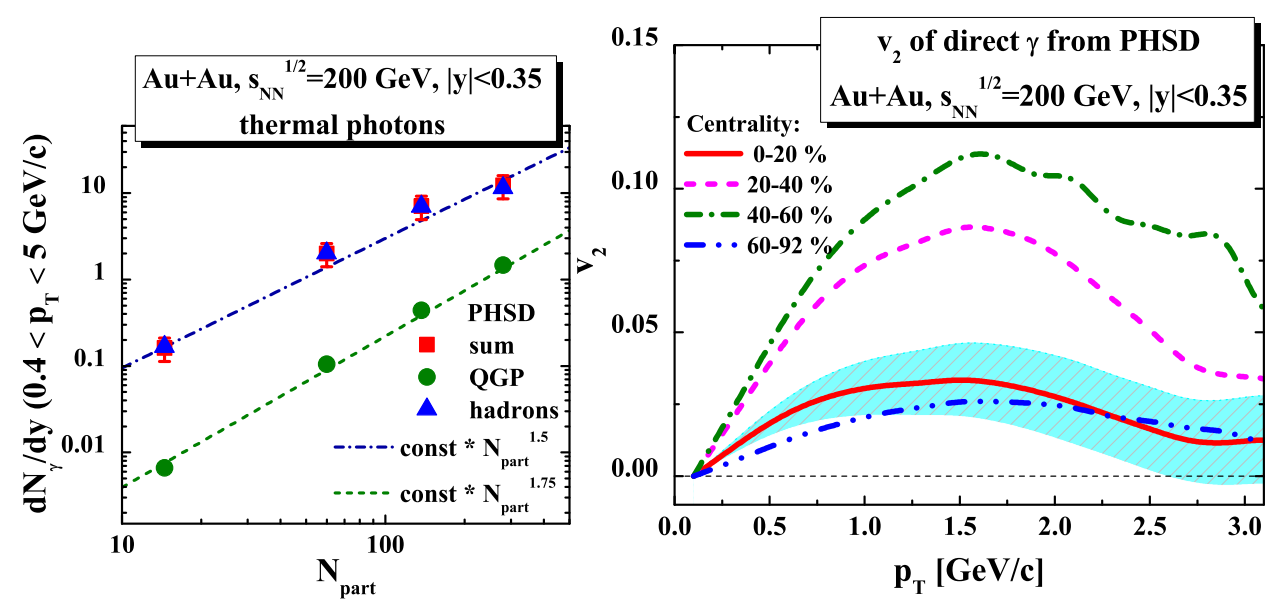

Figure 2. (1.h.s.) Integrated spectra of thermal photons produced in $\mathrm{Au}+\mathrm{Au}$ collisions at $\sqrt{s_{N N}}=200 \mathrm{GeV}$ versus the number of participants $N_{\text {part }}$. The scaling with $N_{\text {part }}$ from the QGP contributions (full dots) and the bresstrahlungs channels (full triangles) are shown separately. (r.h.s.) The elliptic flow $v_{2}\left(p_{T}\right)$ of direct photons produced by binary processes in $\mathrm{Au}+\mathrm{Au}$ collisions at $\sqrt{s_{N N}}=200 \mathrm{GeV}$ for different centralities versus the photon transverse momentum $p_{T}$. The hatched area displays the statistical uncertainty (for the most central bin).

flow results from the interplay of two independent factors: First, the channel decomposition of the direct photon yield changes (cf. Fig. 1): the admixture of photons from the hadronic phase increases for more peripheral collisions. Since the PHSD approach predicts a very small $v_{2}$ of photons produced in the initial hot deconfined phase by partonic channels of the order of $2 \%$ the photon flow $v_{2}$ shows about the same signal for the most central bin. On the other hand, the photons from the hadronic sources show a strong elliptic flow (up to $10 \%$ ), on the level of the $v_{2}$ of final hadrons. Accordingly, since the channel decomposition of the direct photons changes with centrality, the elliptic flow of the direct photons increases with decreasing centrality and becomes roughly comparable with the elliptic flow of pions in peripheral collisions. The elliptic flow in the most peripheral bin is also low in Fig. 2 (r.h.s.) because all the colliding particles have little flow at this high impact parameter $b$.

\section{Dileptons}

\subsection{Production sources}

Dileptons $\left(e^{+} e^{-}\right.$or $\mu^{+} \mu^{-}$pairs) can be emitted from all stages of the reactions as well as a photons. One of the advantages of dileptons compared to photons is an additional 'degree of freedom' - the invariant mass $M$ which allows to disentangle various sources. The following production sources of dileptons in $p+p, p+A$ and $A+A$ collisions are leading:

1) Hadronic sources:

(i) at low invariant masses $(M<1 \mathrm{GeV} c)$ - the Dalitz decays of mesons and baryons $\left(\pi^{0}, \eta, \Delta, \ldots\right)$ and the direct decay of vector mesons $(\rho, \omega, \phi)$ as well as hadronic bremsstrahlung;

(ii) at intermediate masses $(1<M<3 \mathrm{GeV} c)$ - leptons from correlated $D+\bar{D}$ pairs, radiation from multi-meson reactions $\left(\pi+\pi, \pi+\rho, \pi+\omega, \rho+\rho, \pi+a_{1}, \ldots\right)$ - so called ' $4 \pi^{\prime}$ contributions;

(iii) at high invariant masses $(M>3 \mathrm{GeV} c)$ - the direct decay of vector mesons $\left(J / \Psi, \Psi^{\prime}\right)$ and initial 
'hard' Drell-Yan annihilation to dileptons $\left(q+\bar{q} \rightarrow l^{+}+l^{-}\right.$, where $\left.l=e, \mu\right)$.

2) 'thermal' QGP dileptons radiated from the partonic interactions in heavy-ion $(A+A)$ collisions that contribute dominantly to the intermediate masses. The leading processes are the 'thermal' $q \bar{q}$ annihilation $\left(q+\bar{q} \rightarrow l^{+}+l^{-}, \quad q+\bar{q} \rightarrow g+l^{+}+l^{-}\right)$and Compton scattering $\left(q(\bar{q})+g \rightarrow q(\bar{q})+l^{+}+l^{-}\right)$.

\subsection{Transport results from SIS to LHC energies}

At energies around $1 \mathrm{AGeV}$ dileptons have been measured in heavy-ion collisions at the BEVALAC in Berkeley by the DLS Collaboration by more than two decades ago. These data led to the so called 'DLS puzzle' because the DLS dilepton yield in $\mathrm{C}+\mathrm{C}$ and $\mathrm{Ca}+\mathrm{Ca}$ collisions at $1 \mathrm{AGeV}$ in the invariant mass range from 0.2 to $0.5 \mathrm{GeV}$ was about five times higher than the results from different transport models at that time using the 'conventional' dilepton sources such as bremsstrahlung, $\pi^{0}, \eta, \omega$ and $\Delta$ Dalitz decays and direct decay of vector mesons $(\rho, \omega, \phi)$ [30]. To solve this puzzle was one of the main motivations to build the HADES (High Acceptance Dilepton Spectrometer) detector at GSI [31]. Indeed the HADES Collaboration could confirm the DLS measurements at $1 \mathrm{AGeV}$ when passing their events for $\mathrm{C}+\mathrm{C}$ through the DLS filter [32]. From the theory side it was argued that the pn bremsstrahlung channel should be sizeably enhanced as compared to the early soft photon calculations [30]. Indeed, a good reproduction of various spectra a different energies could be achieved within the HSD calculations in Ref. [33]. Note, however, that even the bremsstrahlung from pn reactions at these low energies is discussed controversally in the community and not available experimentally. We here report on the actual status of the transport calculations in comparison to the HADES data [34].

Fig. 3 (l.h.s.) shows the mass differential dilepton spectra - normalized to the $\pi^{0}$ multiplicity from $\mathrm{HSD}$ calculations for $\mathrm{C}+\mathrm{C}$ at $2 \mathrm{AGeV}$ in comparison to the HADES data [35]. The theoretical calculations passed through the corresponding HADES acceptance filters and mass/momentum resolutions which leads to a smearing of the spectra at high invariant mass and particularly in the $\omega$ peak region. The upper part shows the case of 'free' vector-meson spectral functions while the lower part presents the result for the 'collisional $\rho$ broadening' scenario. Here the difference between in-medium scenarios is of minor importance and partly due to the limited mass resolution which smears out the spectra. Fig. 3 (r.h.s.) displays the mass differential dilepton spectra - normalized to the $\pi^{0}$ multiplicity - from HSD calculations for the heavier system $\mathrm{Ar}+\mathrm{KCl}$ at $1.76 \mathrm{AGeV}$ in comparison to the HADES data [31]. The upper part shows again the case of 'free' vector-meson spectral functions while the lower part gives the result for the 'collisional broadening' scenario. Also in this data set the enhancement around the $\rho$ mass is clearly visible. For the heavier system the 'collisional broadening' scenario shows a slightly better agreement with experiment than the 'free' result and we expect that for larger systems the difference between the two approaches increases. We note that with increasing mass $\mathrm{A}+\mathrm{A}$ of the system the low mass dilepton regime from roughly 0.15 to $0.5 \mathrm{GeV}$ increases due to multiple $\Delta$-resonance production and Dalitz decay. The dileptons from intermediate $\Delta$ 's, which are part of the reaction cycles $\Delta \rightarrow \pi N ; \pi N \rightarrow \Delta$ and $N N \rightarrow N \Delta ; N \Delta \rightarrow N N$, escape from the system while the decay pions do not [34]. With increasing system size more generations of intermediate $\Delta$ 's are created and the dilepton yield enhanced accordingly. In inclusive $\mathrm{C}+\mathrm{C}$ collisions there is only a moderate enhancement relative to scaled $p+p$ and $p+n$ collisions due to the small size of the system while in $\mathrm{Ar}+\mathrm{KCl}$ reactions already several (3-4) reactions cycles become visible. On the other hand the effects from a broadened vector-meson spectral function is barely visible for both systems and calls for $\mathrm{Au}+\mathrm{Au}$ collisions. Indeed, the respective data have been taken and are currently analyzed. For detailed predictions we refer the reader to Ref. [34].

Dileptons from heavy-ion collisions at SPS energies have been measured in the last decades by the CERES [36] and NA60 [37] Collaborations. The high accuracy dimuon NA60 data provide a unique possibility to subtract the hadronic cocktail from the spectra and to distinguish different 

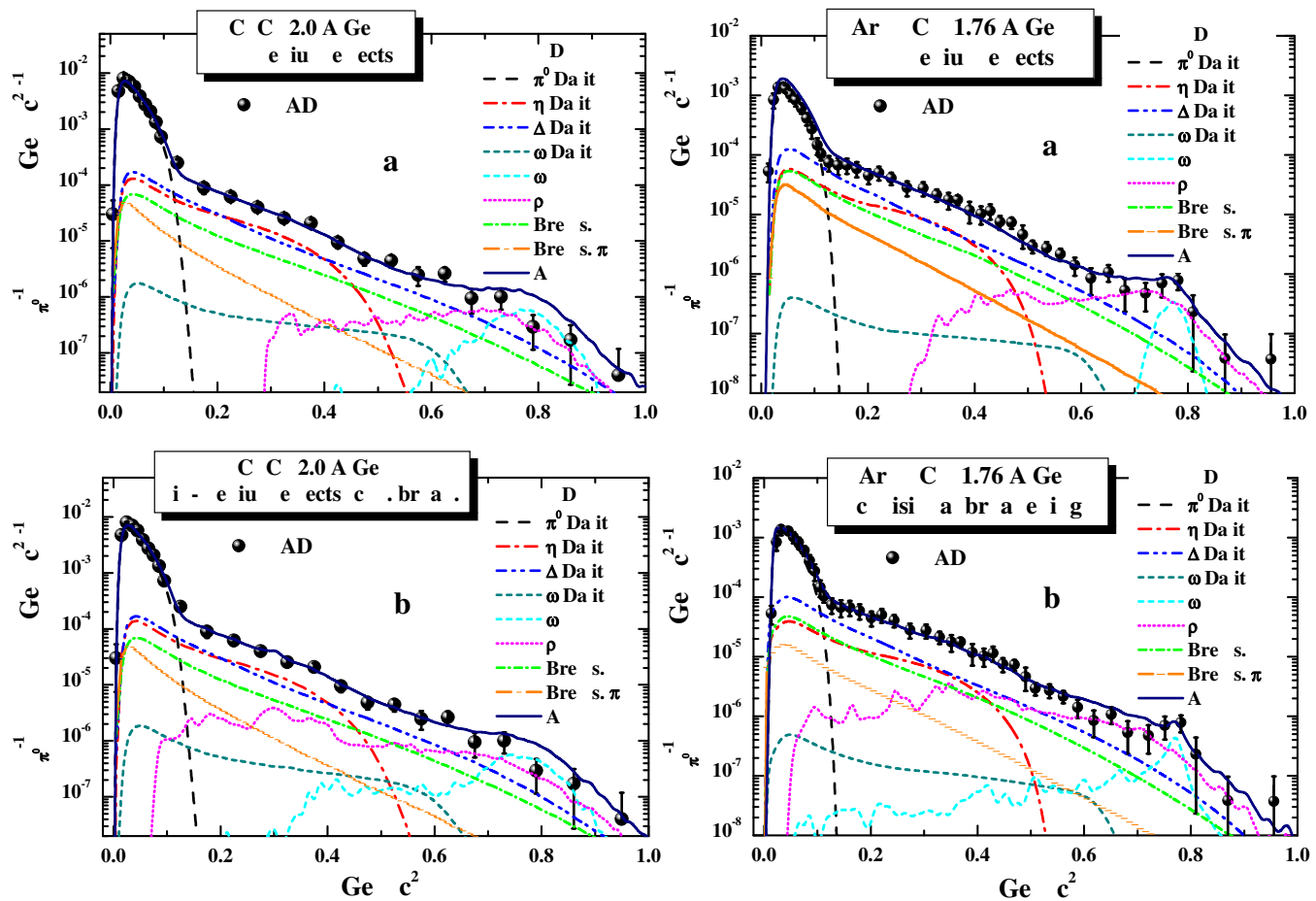

Figure 3. The mass differential dilepton spectra - normalized to the $\pi^{0}$ multiplicity - from HSD calculations for $\mathrm{C}+\mathrm{C}$ at $2 \mathrm{AGeV}$ (1.h.s.) and $\mathrm{Ar}+\mathrm{KCl}$ at $1.76 \mathrm{AGeV}$ (r.h.s.) in comparison to the HADES data [31, 35]. The upper parts (a) shows the case of 'free' vector-meson spectral functions while the lower parts (b) give the result for the 'collisional broadening' scenario. The different colour lines display individual channels in the transport calculation (see legend). The theoretical calculations passed through the corresponding HADES acceptance filter and mass/momentum resolutions.

in-medium scenarios for the $\rho$-meson spectral function such as a collisional broadening and dropping mass $[2,38]$. The main messages obtained by a comparison of the variety of model calculations (see e.g. $[2,39,40])$ with experimental data can be summarized as

(i) the low mass spectra $[36,37]$ provide a clear evidence for the collisional broadening of the $\rho$-meson spectral function in the hot and dense medium;

(ii) the intermediate mass spectra above $M>1 \mathrm{GeV} / c^{2}$ [37] are dominated by partonic radiation;

(iii) the rise and fall of the inverse slope parameter of the dilepton $p_{T}$-spectra (effective temperature) $T_{\text {eff }}$ [37] provide evidence for the thermal QGP radiation;

(iv) isotropic angular distributions [37] are an indication for a thermal origin of dimuons.

An increase in energy from SPS to RHIC has opened new possibilities to probe by dileptons a possibly different matter at very high temperature, i.e. dominantly in the QGP stage, created in central heavy-ion collisions. The dileptons $\left(e^{+} e^{-}\right.$pairs) have been measured first by the PHENIX Collaboration for $p p$ and $A u+A u$ collisions at $\sqrt{s}=200 \mathrm{GeV}$ [41]. A large enhancement of the dilepton yield relative to the scaled $p p$ collisions in the invariant mass regime from 0.15 to $0.6 \mathrm{GeV} / c^{2}$ 

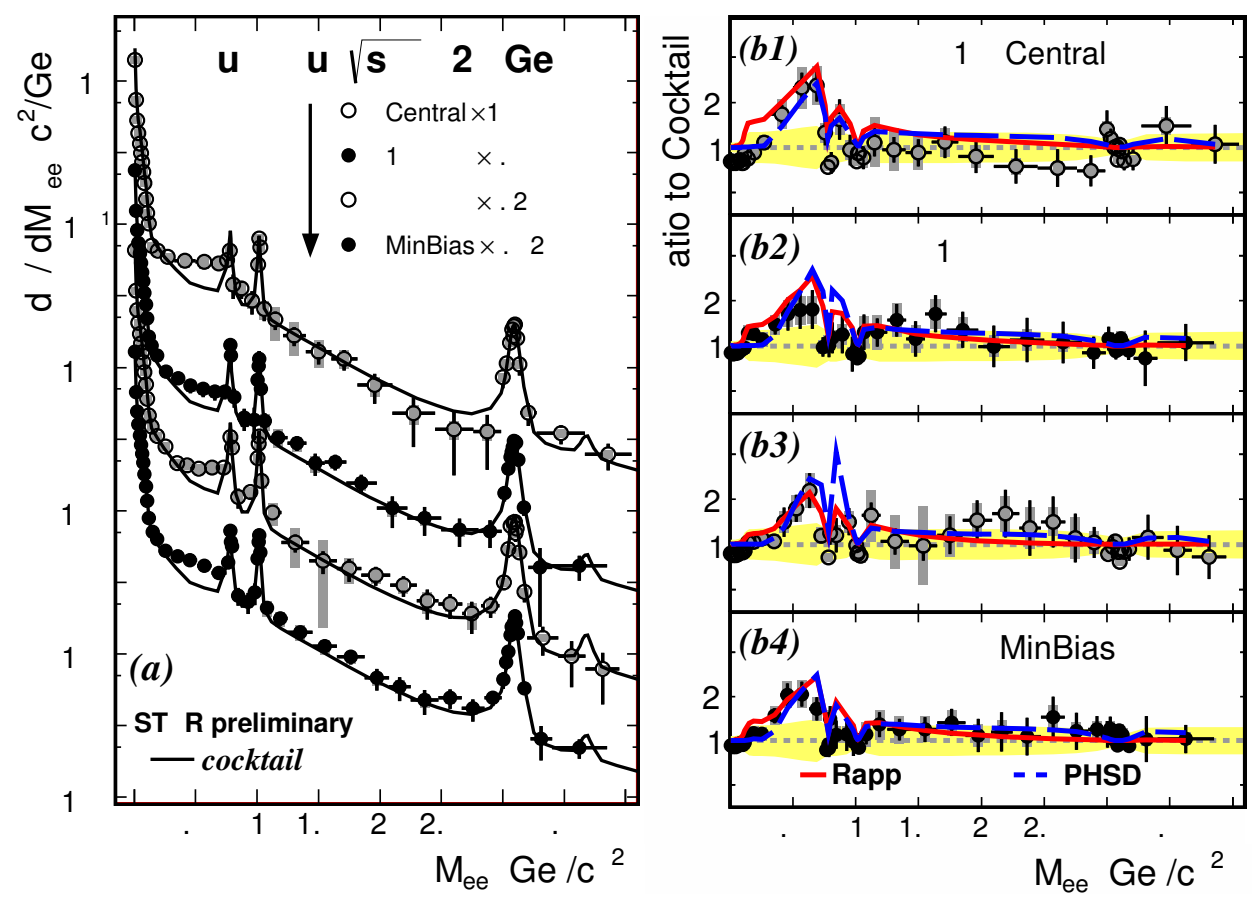

Figure 4. Centrality dependence of the midrapidity dilepton yields (left) and its ratios (right) to the 'cocktail' for $0-10 \%, 10-40 \%, 40-80 \%, 0-80 \%$ central Au+Au collisions at $\sqrt{s}=200 \mathrm{GeV}$ : a comparison of STAR data with theoretical predictions from the PHSD ('PHSD' - dashed lines) and the expanding fireball model ('Rapp' - solid lines). The figure is taken from Ref. [46].

has been reported for central $\mathrm{Au}+\mathrm{Au}$ reactions. This observation has stimulated a lot of theoretical activity (see the model comparison with the data in Ref. [41]). The main messages - which hold up-to-now - can be condensed such that the theoretical models, which provide a good description of $p p$ dilepton data and peripheral $A u+A u$ data, fail in describing the excess in central collisions even with in-medium scenarios for the vector-meson spectral function [39]. The missing strengths might be attributed to low $p_{T}$ sources [42]. On the other hand the intermediate mass spectra are dominated by the QGP radiation as well as leptons from correlated charm pairs $(D+\bar{D})[39,42,43]$.

In this respect it is very important to have independent measurements which have been carried out by the STAR Collaboration [44]. Fig. 4 shows the comparison of STAR data of midrapidity dilepton yields (1.h.s.) and its ratios (r.h.s.) to the 'cocktail' for $0-10 \%, 10-40 \%, 40-80 \%, 0-80 \%$ central $\mathrm{Au}+\mathrm{Au}$ collisions at $\sqrt{s_{N N}}=200 \mathrm{GeV}$ in comparison to the theoretical model predictions from the PHSD approach and the expanding fireball model of Rapp and collaborators. As seen from Fig. 4 the excess of the dilepton yield over the expected cocktail is larger for very central collisions and consistent with the model predictions including the collisional broadening of the $\rho$-meson spectral function at low invariant mass and QGP dominated radiations at intermediate masses. Moreover, the recent STAR dilepton data for Au+Au collisions from the Beam Energy Scan (BES) program for $\sqrt{s_{N N}}=19.6,27,39$ and $62.4 \mathrm{GeV}[21,45,46]$ are also in line with the expanding fireball model (as well as PHSD) predictions with a $\rho$ collisional broadening [46]. According to the PHSD calculations 

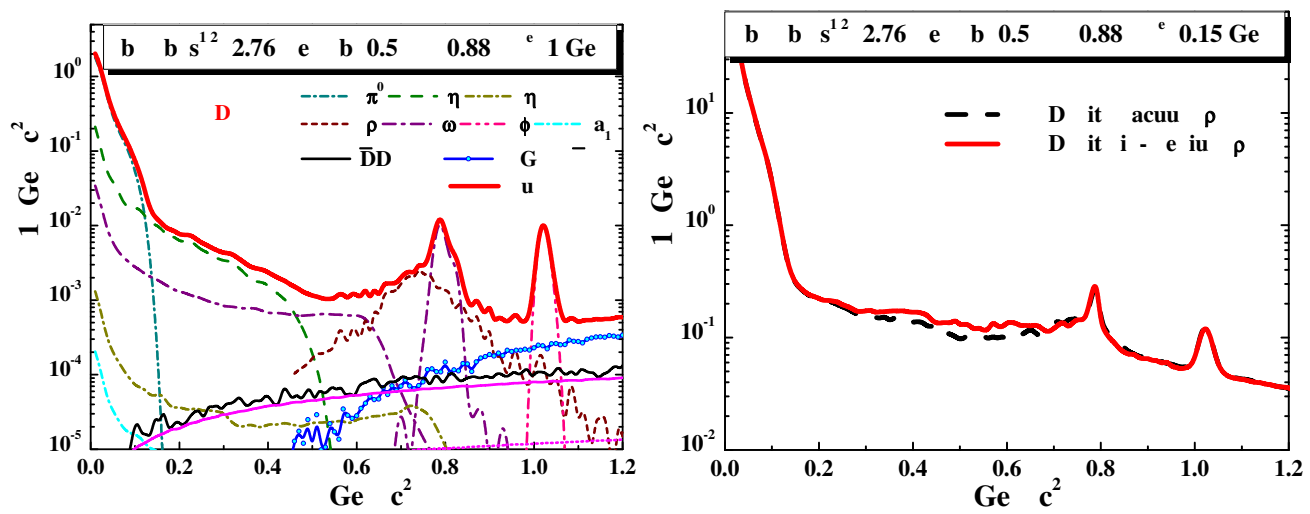

Figure 5. Midrapidity dilepton yields for $\mathrm{Pb}+\mathrm{Pb}$ at $\sqrt{s_{N N}}=2.76 \mathrm{TeV}$ (1.h.s.) for a lepton $p_{T}$ cut of $1 \mathrm{GeV} / \mathrm{c}$. The channel decomposition is explained in the legend. (r.h.s.) Same as for the l.h.s. but for a lepton $p_{T}$ cut of $0.15 \mathrm{GeV} / \mathrm{c}$ for a 'free' $\rho$ spectral function (dashed line) and the collisional broadening scenario (solid line). The figures are taken from Ref. [48].

the excess is increasing with decreasing energy due to a longer $\rho$-propagation in the high baryon density phase (see Fig. 3 in [21]).

The upcoming PHENIX data for central Au+Au collisions - obtained after an upgrade of the detector - together with the BES-II RHIC data should provide finally a consistent picture on the low mass dilepton excess in relativistic heavy-ion collisions. On the other hand, the upcoming ALICE data [47] for heavy-ion dileptons for $\mathrm{Pb}+\mathrm{Pb}$ at $\sqrt{s}=2.76 \mathrm{TeV}$ will give a clean access to the dileptons emitted from the QGP [43, 48]. In Fig. 5 (1.h.s.) we present the PHSD predictions for central $\mathrm{Pb}+\mathrm{Pb}$ collisions [48] in the low mass sector for a realistic lepton $p_{T}$ cut of $1 \mathrm{GeV} / \mathrm{c}$. It is clearly seen that the QGP sources and contribution from correlated $D \bar{D}$ pairs are subleading in the low mass regime where we find the conventional hadronic sources. For a lepton $p_{T}$ cut of $1 \mathrm{GeV} / \mathrm{c}$ (1.h.s.) one practically cannot identify an effect of the $\rho$ collisional broadening in the dilepton spectra in the PHSD calculations. Only when applying a low $p_{T}$ cut of $0.15 \mathrm{GeV} / \mathrm{c}$ a small enhancement of the dilepton yield from 0.3 to $0.7 \mathrm{GeV}$ becomes visible (r.h.s. of Fig. 5). This low sensitivity to hadronic in-medium effects at LHC energies is due to the fact that the hadrons come out late in central $\mathrm{Pb}+\mathrm{Pb}$ collisions and are boosted to high velocities due to the high pressure in the early partonic phase.

In the end, we mention that promising perspectives with dileptons have been suggested in Ref. [49] to measure the anisotopy coefficients $v_{n}, n=2,3$ similar to photons. The calculations with the viscous (3+1)d MUSIC hydro for central Au+Au collisions at RHIC energies show that $v_{2}, v_{3}$ are sensitive to the dilepton sources and to the EoS and $\eta / s$ ratio. The main advantage of measuring flow coefficients $v_{n}$ with dileptons compared to photons is the fact that an extra degree of freedom $M$ might allow to disentangle the sources.

\section{Conclusions}

In conclusion, our calculations show that the photon production in the QGP is dominated by the early phase (similar to hydrodynamic models) and is localized in the center of the fireball, where the collective flow is still rather low, i.e. on the $2-3 \%$ level, only. Thus, the strong $v_{2}$ of direct photons 
- which is comparable to the hadronic $v_{2}$ - in PHSD is attributed to hadronic channels, i.e. to meson binary reactions, meson-meson and meson-baryon bremsstrahlung which are not subtracted in the data. On the other hand, the strong $v_{2}$ of the 'parent' hadrons, in turn, stems from the interactions in the QGP. We have argued that a precise measurement of the centrality dependence of the elliptic flow of direct photons together with their differential spectra should help in clarifying the the photon $v_{2}\left(p_{T}\right)$ "puzzle". Note however, that the hadronic bremsstrahlungs channels are not well under control and our present results should be taken as 'upper limits'. Some more work will have to be done in this direction.

The main messages from our dilepton campaign may be formulated as follows: i) the low mass $\left(M=0.2-0.6 \mathrm{GeV} / c^{2}\right)$ dilepton spectra show sizable changes due to hadronic in-medium effects, i.e. multiple hadronic resonance formation (at SIS energies) or a modification of the properties of vector mesons (such as collisional broadening) in the hot and dense hadronic medium (partially related to chiral symmetry restoration); these effects can be observed at all energies up to LHC (preferentially in heavy systems) but are most pronounced in the FAIR/NICA energy regime; (ii) at intermediate masses the QGP ( $q \bar{q}$ thermal radiation) dominates for $M>1.2 \mathrm{GeV} / c^{2}$, it grows with increasing energy and becomes dominant at the LHC energies. The dilepton measurements within the future experimental energy and system size scan ( $p p, p A, A A)$ from low to top RHIC energies as well as new ALICE data at LHC energies will extend our knowledge on the properties of hadronic and partonic matter via its electromagnetic radiation.

The authors acknowledge financial support through the 'HIC for FAIR' framework of the 'LOEWE' program and like to thank all their coauthors for their help and valuable contributions.

\section{References}

[1] E.L. Feinberg, Izv. Akad. Nauk Ser. Fiz. 34, 1987 (1970); Nuovo Cim. A34, 391 (1976) ; E.V. Shuryak, Phys. Lett. B78, 150 (1978).

[2] R. Rapp, Adv. High Energy Phys. 2013, 148253 (2013). R. Rapp, J. Wambach, H. van Hees, in "Relativistic Heavy-Ion Physics", edited by R. Stock, Landolt-Boernstein, Volume I/23, 4-1 (2010).

[3] A. Adare et al. (PHENIX Collaboration), Phys. Rev. 81, 034911 (2010).

[4] W. Cassing and E.L. Bratkovskaya, Phys. Rev. C 78, 034919 (2008); Nucl. Phys. A 831, 215 (2009); E.L. Bratkovskaya et al., Nucl. Phys. A 856, 162 (2011).

[5] L. D. McLerran and T. Toimela, Phys. Rev. D31, 545 (1985).

[6] C. Gale and J. Kapusta, Phys. Rev. C 35, 2107 (1987).

[7] R. Rapp, G. Chanfray, and J. Wambach, Nucl. Phys. A617, 472 (1997).

[8] W. Cassing et al., Phys. Rev. Lett. 110, 182301 (2013).

[9] K. Kajantie, J. Kapusta, L. McLerran, and A. Mekjian, Phys. Rev. D 34, 2746 (1986).

[10] D. Cabrera et al., arXiv:1304.2309.

[11] P. B. Arnold, G. D. Moore, and L. G. Yaffe, JHEP 0111, 057 (2001).

[12] J. Ghiglieri, arXiv:1407.8470[hep-ph].

[13] J. I. Kapusta, P. Lichard, and D. Seibert, Phys.Rev. D44, 2774 (1991).

[14] E. L. Bratkovskaya, S. M. Kiselev and G. B. Sharkov, Phys. Rev. C 78, 034905 (2008).

[15] O. Linnyk et al., Phys. Rev. C88, 034904 (2013); ibid C89, 034908 (2014).

[16] S. Turbide, R. Rapp, and C. Gale, Phys. Rev. C69, 014903 (2004).

[17] R. Albrecht et al. (WA98 Collaboration), Phys. Rev. Lett. 76, 3506 (1996); M.M. Aggarval et al., Phys. Rev. Lett. 85, 3595 (2000); ibid 93, 022301 (2004). 
[18] W. Liu and R. Rapp, Nucl. Phys. A 96, 101 (2007).

[19] A. Adare et al. (PHENIX Collaboration), Phys. Rev. Lett. 109, 122302 (2012).

[20] D. Lohner et al. (ALICE Collaboration), J. Phys. Conf. Ser. 446, 012028 (2013); M. Wilde et al., Nucl. Phys. A 904-905, 573c (2013).

[21] L. Ruan, arXiv:1407.8153[nucl-ex].

[22] F. Bock (for the ALICE Collaboration), proceedings of QM2014.

[23] U. Heinz, J. Liu, and C. Shen, arXiv:1403.8101 [nucl-th]

[24] W. Cassing and E.L. Bratkovskaya, Phys. Rept. 308, 65 (1999).

[25] W. Cassing, Eur. Phys. J. ST 168, 3 (2009).

[26] O. Linnyk, J. Phys. G 38, 025105 (2011).

[27] A. Adare et al. (PHENIX Collaboration), arXiv:1405.3940 [nucl-ex].

[28] S. Mizuno (for the PHENIX Collaboration), proceedings of QM2014.

[29] C. Shen et al., Phys. Rev. C 89, 044910 (2014).

[30] E. L. Bratkovskaya and C. M. Ko, Phys. Lett. B 445, 265 (1999).

[31] G. Agakishiev et al. [HADES Collaboration], Phys. Rev. C 84, 014902 (2011).

[32] G. Agakishiev et al. [HADES Collaboration], Phys. Lett. B 663, 43 (2008).

[33] E. L. Bratkovskaya and W. Cassing, Nucl. Phys. A 807, 214 (2008).

[34] E. L. Bratkovskaya et al., Phys. Rev. C 87, 064907 (2013)

[35] G. Agakishiev et al. [HADES Collaboration], Phys. Lett. B 690, 118 (2010).

[36] D. Adamova et al. (CERES Collaboration), Nucl. Phys. A 715, 262 (2003); Phys. Lett. B 666, 425 (2008).

[37] R. Arnaldi et al. (NA60 Collaboration), Phys. Rev. Lett. 96, 162302 (2006); Eur. Phys. J. C 61, 711 (2009).

[38] G. Q. Li, C. M. Ko and G. E. Brown, Phys. Rev. Lett. 75, 4007 (1995).

[39] E. L. Bratkovskaya, W. Cassing, and O. Linnyk, Phys. Lett. B670, 428 (2009). O. Linnyk et al., Phys. Rev. C 84, 054917 (2011).

[40] J. Ruppert and T. Renk, Phys. Rev. C 71, 064903 (2005); Erratum-ibid. C 75, 059901 (2007); K. Dusling, D. Teaney, and I. Zahed, Phys. Rev. C 75, 024908 (2007); E. Santini et al., Phys. Rev. C 84, 014901 (2011).

[41] A. Toia et al. (PHENIX Collaboration), Nucl. Phys. A 774, 743 (2006); J. Phys. G 35, 104037 (2008); A. Adare et al., Phys. Lett. B670, 313 (2009); Phys. Rev. C 81, 034911 (2010).

[42] O. Linnyk et al., Phys. Rev. C 85, 024910 (2012).

[43] R. Rapp, Adv. High Energy Phys. 2013, 148253 (2013).

[44] L. Ruan et al. (STAR Collaboration), Nucl. Phys. A 855, 269 (2011).

[45] X. Dong et al. (STAR Collaboration), Nucl. Phys. A 904-905, 19c (2013); F. Geurts et al., Nucl. Phys. A 904-905, 217c (2013).

[46] P. Huck (for the STAR Collaboration), proceedings QM2014.

[47] M. K. Köhler (for the ALICE Collaboration), proceedings QM2014.

[48] O. Linnyk et al., Phys.Rev. C 87, 014905 (2013).

[49] G. Vujanovic et al., Phys. Rev. C 89, 034904 (2014); arXiv:1408.1098. 